\title{
Intersections
}

Canadian Journal of Music

Revue canadienne de musique

Musiques. Une Encyclopédie pour le XXI $I^{e}$ siècle. 2007. « 5. L'Unité de la musique ", sous la direction de Jean-Jacques Nattiez. Arles-Paris : Actes Sud/Cité de la musique, 1253 p., ISBN 978-2-7427-6974-2 (couverture rigide)

\section{Jessica Roda}

Volume 28, numéro 2, 2008

URI : https://id.erudit.org/iderudit/029957ar

DOI : https://doi.org/10.7202/029957ar

Aller au sommaire du numéro

Éditeur(s)

Canadian University Music Society / Société de musique des universités canadiennes

ISSN

1911-0146 (imprimé)

1918-512X (numérique)

Découvrir la revue

Citer ce compte rendu

Roda, J. (2008). Compte rendu de [Musiques. Une Encyclopédie pour le XXI siècle. 2007. « 5. L'Unité de la musique ", sous la direction de Jean-Jacques

Nattiez. Arles-Paris : Actes Sud/Cité de la musique, 1253 p., ISBN

978-2-7427-6974-2 (couverture rigide)]. Intersections, 28(2), 96-104.

https://doi.org/10.7202/029957ar

Tous droits réservés (C) Canadian University Music Society / Société de musique des universités canadiennes, 2009
Ce document est protégé par la loi sur le droit d'auteur. L'utilisation des services d'Érudit (y compris la reproduction) est assujettie à sa politique d'utilisation que vous pouvez consulter en ligne.

https://apropos.erudit.org/fr/usagers/politique-dutilisation/ 


\section{BOOK REVIEWS / RECENSIONS}

Musiques. Une Encyclopédie pour le XXI siècle. 2007. " 5. L'Unité de la musique », sous la direction de Jean-Jacques Nattiez. Arles-Paris : Actes Sud/Cité de la musique, 1253 p., ISBN 978-2-7427-6974-2 (couverture rigide).

L'aventure de l'Encyclopédie pour le XXI siècle, dont Jean-Jacques Nattiez, professeur titulaire à la Faculté de musique de l'Université de Montréal, est le maître d'ouvre, s'est achevée en septembre 2007 avec la parution de l'édition française de son cinquième volume. Son sous-titre provocateur, «L'Unité de la musique », laisse rêveur bon nombre de musiciens et de musicologues, d'autant plus qu'il renforce l'idée d'Une Encyclopédie pour le XXIe siècle. En effet, Nattiez clôture ce volet final de façon grandiose en offrant des réflexions sur les problématiques actuelles et celles qu'il est possible d'envisager pour le futur.

Ce livre est une synthèse et un approfondissement de l'ensemble des objets étudiés dans les volumes précédents. Par ailleurs, il ajoute une nouveauté en intégrant la problématique des musiques hybrides qui suscite une "interrogation musicologique de l'âge post-moderne » (p. 21). Le musical y est analysé comme un phénomène planétaire où l'on tente de saisir si, au-delà de la diversité des manifestations de la musique dans l'histoire et dans les cultures, elles ne se rapportent pas à un phénomène unique tel que LA musique (au singulier), qui constituerait leur commune immanence.

Lachèvement de ce projet encyclopédique s'est réalisé par l'écriture de 43 articles rédigés par des musicologues provenant des cinq continents. En partant de la diversité musicale pour aller vers son unité, l'organisation de ce volume a été minutieusement réfléchie afin de conduire progressivement le lecteur vers l'exigence des universaux et des "vérités locales » (p. 1208). Pour une compréhension générale du cheminement intellectuel et du contenu présenté dans ce volume, nous recommandons à tout lecteur de se pencher sur l'article de présentation de Nattiez, "Éclatement ou unité de la musique » (p. 17-32), qui témoigne d'un éminent esprit de synthèse. Par l'accroche «Un volume qui déplaira ", c'est avec audace qu'il met en garde le lecteur puriste ou dubitatif face à une approche globale de la musique. En décrivant le paysage musical actuelsubmergé par les processus d'échange et de mondialisation des phénomènes musicaux (chapitre 1) - Nattiez propose l'idée que «si une musique peut être arrachée à son contexte d'origine et acceptée ailleurs, c'est bien qu'il y a quelque chose d'universel en elle [...], quelque chose, dans sa nature sémiologique, qui explique la possibilité de sa circulation transculturelle et planétaire» (p. 25). Les questions théoriques relatives aux universaux, à l'unité et à la conception du musical feront l'objet d'une discussion plus loin, notamment lorsqu'il sera 
question des considérations théoriques développées dans l'article de Molino et Nattiez.

Étant donné l'impossibilité de rendre compte de l'intégralité du volume, nous avons choisi de nous consacrer surtout à la première partie, qui apparaît comme le cadre conceptuel qui sous-tend l'organisation de cet ultime volume. Cela ne nous empêchera pas de faire allusion à des articles présentés dans les autres parties et qui, au besoin, éclaireront la matière recensée. Nous nous pencherons bien évidemment sur la question des universaux, qui est au cœur du projet encyclopédique.

\section{Contenu et PROJet global}

Le cinquième volume de l'Encyclopédie comprend quatre parties. La première, intitulée "L'échange des cultures ", décrit les allers-retours culturels entre des musiques éloignées dans l'espace et dans le temps. Une subdivision en « Musique et colonialisme » et "L'expansion mondiale des musiques traditionnelles et populaires » a été adoptée pour distinguer la relation dialectique établie entre les musiques occidentales dites savantes et les autres musiques - cette dernière désignant ce qu'il est convenu d'appeler les musiques dites traditionnelles et populaires.

Après avoir plaidé pour la recherche des universaux et de l'unité de la musique ("Typologies et Universaux », Jean Molino et Jean-Jacques Nattiez, p. 337-396), il s'agit dans la seconde partie du volume de répondre à la question "Qu'est-ce que la musique? » à travers une triple perspective anthropologique, ethnomusicologique et historique. Le problème de «L'oral et l'écrit » est également abordé en interrogeant la définition de l'oralité, la transmission orale et écrite ou le rôle de la notation musicale. En prenant le contre-pied des idées reçues, Ignazio Macchiarella ("Le faux-bourdon entre oralité et écriture», p. 528549) et Peter G. Jeffery ( La transmission orale et écrite : l'exemple du chant byzantin ", p. 550-576) montrent que la scission entre musiques occidentales écrites et musiques de tradition orale n'est pas si radicale, et qu'au contraire les échanges y sont multiples. Pour répondre aux orientations relativement récentes de la musicologie telles que les nouveaux mécanismes de diffusion et d'apprentissage de la musique, dans un article saisissant intitulé "Qu'est-ce que l'oralité musicale ? » (p. 477-527), Jean Molino propose le concept d'« auralité ». Ce concept est un néologisme de l'anglais (aural, lié à l'audition) dont l'objectif est de souligner l'importance de l'écoute sur les nouveaux mécanismes.

D'autres auteurs accordent de l'importance à tout ce qui va au-delà de la partition, notamment en ce qui a trait à la création, à la réception et à l'interaction entre ces deux processus (i.e. écriture et oralité). Mentionnons l'article d'AnneMarie Green, «L'influence de l'espace sur la réception musicale (p. 727-742), où la sociologue s'intéresse au fonctionnement de la société contemporaine à partir des pratiques de réception musicale que mettent en jeu le baladeur ou des lieux comme le métro et la rue. Dans sa réflexion, elle propose une approche novatrice de l'espace musical qui tend vers l'étude des nouveaux espaces comme élément de "réenchantement » du monde, plutôt que comme fond sonore non «légitimé » (p. 741). L'article novateur de Philip Tagg, « Significations 
musicales dans les musiques classiques et populaires » (p. 743-772), analyse l'expression musicale de l'angoisse dans plus d'une vingtaine de compositions à partir de la théorie des "musèmes " (Seeger, 1960), qui permet selon Tagg de comprendre ce que la musique signifie au-delà d'elle-même.

Ce n'est que dans la troisième partie que la tentative de catégorisation et d'unification s'établit. Les «Typologies et comparaisons " dans l'analyse des "Voix et instruments » sont au cœur de la problématique étudiée ici, ainsi que la question des « Rythmes, échelles, mélodies, polyphonies » dans le but de définir des universaux tangibles. Un essai de typologie sur l'organisation du temps musical est proposé par l'ethnomusicologue Simha Arom ("L'organisation du temps musical : essai de typologie ", p. 927-944). Il y développe l'idée selon laquelle la structuration du fait musical résulte d'une combinatoire de concepts et de principes à l'œuvre dans toutes les cultures. Étant donné son orientation structuraliste, il n'est pas surprenant de constater qu'un certain nombre de chercheurs sympathiques à ses idées, tels Nathalie Fernando, Susanne Fürniss ou Olivier Tourny soient insérés dans ce chapitre.

En guise de conclusion, une quatrième partie est consacrée aux « Problèmes contemporains de la connaissance du musical » où les notions d'authenticité, d'identité et d'esthétique sont discutées dans le but de réexaminer nos modes de connaissance de la musique. Le volume s'achève par un article audacieux où Nattiez réaffirme sa position : l'élimination des barrières idéologiques par l'ouverture à la musique de toutes les époques et de toutes les cultures. Pour l'auteur, l'unité de la musique permettra de comprendre le fait musical dans sa globalité, et cela par le biais d'une musicologie générale. Cette dernière doit intégrer l'ensemble des méthodes et des savoirs. Ainsi nous conduira-t-elle peu à peu vers l'unité de la musicologie, dont l'organisation repose sur la construction de typologies. En établissant des comparaisons entre les objets, le chercheur peut ainsi tenir compte des points communs et mieux les intégrer dans une même catégorie. Selon Nattiez, la diversité serait un préalable à l'unité.

Sur le plan du contact entre les cultures, les articles de Kofi Agawu, Gérard Béhague et François Picard décrivent l'impact du colonialisme sur les sociétés musicales africaine, latino-américaine et asiatique (respectivement " L'impact du colonialisme sur la musique africaine », p. 37-67; " La conquête coloniale et l'implantation de la musique européenne en Amérique latine », p. 103-127; "L'implantation de la musique européenne en Asie orientale ", p. 128-152). Agawu met l'accent sur les transformations de la musique africaine face à la colonisation, tandis que Béhague et Picard se placent davantage du côté de la musique européenne en examinant comment elle s'est implantée dans divers pays. Ces trois essais s'intéressent au contact entre les cultures « étrangères » et locales, ainsi qu'à la coexistence des deux modes d'expression, aux transformations du modèle des autochtones ou à l'absence d'influences qui en résultent.

Larticle de Kofi Agawu est ambitieux en ce qu'il propose de transformer les discours sur la musique africaine par l'entremise d'une théorie qui rejetterait la comparaison entre éléments européens et africains. Cela doit servir à explorer et à comprendre de façon plus exhaustive les situations de contact. De plus, l'auteur se prononce sur le désir d'envisager la musique africaine contemporaine 
comme grande richesse de créativité, plutôt que comme nostalgie du passé précolonial.

Béhague et Picard offrent une brillante synthèse historique des processus d'interaction rencontrés en Amérique latine (Pérou, Colombie, Brésil, Mexique) et en Asie orientale (Chine, Corée, Japon). Picard décrit ces processus de façon diachronique en montrant les événements marquants qui ont provoqué rejets, adaptations ou échecs dans cette rencontre entre Orient et Occident. Contrairement à l'Afrique ou à l'Amérique latine, où les substitutions du langage musical occidental sont respectivement partielles et totales, les pratiques musicales asiatiques ne seront pas considérées comme fusion, mais comme « reconnaissance mutuelle grandissante [...], débouchant sur des compétences cumulées ou croisées où les racines et les histoires des uns et des autres sont désormais entremêlées » (p. 149).

Les articles de Jean-Pierre Bartoli ("Orientalisme et exotisme de la Renaissance à Debussy », p. 155-181) et Susanna Pasticci ("L'influence des musiques non européennes sur la musique occidentale du XXe siècle », p. 182-203) tentent une synthèse historique qui se déplace des prémisses de l'intérêt pour les musiques traditionnelles et populaires à la naissance de l'ethnomusicologie. Dans une approche théorique, Pasticci aborde les questions animant l'esprit des musiciens des XIX ${ }^{\mathrm{e}}$ et $\mathrm{XX}^{\mathrm{e}}$ siècles, dont celles du mythe de l'étranger, de l'identité et de l'altérité. En expliquant que les musiques non occidentales et extra-européennes jouent un rôle social en organisant la vie quotidienne, l'auteure néglige celui de la musique occidentale savante (p. 184). Pourtant, dans son article "L'interaction des musiques et du public dans l'invention musicale » (p. 712726), Regula Burckhardt Qureshi démontre à travers une analyse pointue que le concert savant occidental est également tributaire d'un rôle social.

Répondant fidèlement au souhait de pluralisme des phénomènes d'échange entre les cultures, plusieurs des articles de cette première partie s'intéressent à la diffusion, à la circulation et à la valorisation des musiques à travers le monde.

Dans « Musique classique, musique populaire et musique du monde sur Internet » (p. 266-285), Sylvia L'Ecuyer constate que ce nouveau moyen de diffusion a transformé notre utilisation, appréhension et compréhension du musical. Comparativement à la radio et au disque, elle explique qu'avec la révolution entamée par Internet, le schisme entre producteurs et consommateurs se résorbe peu à peu en laissant place à un nouvel ordre musical centré sur les technologies de la communication. Y est discuté le problème du droit à la propriété intellectuelle et musicale qui intéresse des chercheurs tels que Pierre-Michel Menger (2001) et Paul Théberge (1997). Lauteure décrit plusieurs moyens mis en place pour protéger ce droit, mais elle prend acte que, jusqu'à ce jour, l'outil infaillible rêvé est difficilement concevable. Dans un contexte où la diffusion et la valorisation ne seront plus entre les mains des grands producteurs, les musiques du monde et les musiques classiques arriveront-elles un jour à trouver un public aussi important que celui des musiques populaires ? C'est un enjeu essentiel pour le XXIe siècle musical.

Au sujet de l'expansion des musiques traditionnelles, dans « La diffusion du didjeridu en Australie et vers le monde occidental» (p. 218-245), Robin Ryan 
démontre que la décontextualisation de l'instrument a engendré d'inévitables changements dans ses significations spirituelles et culturelles. Le didjeridu "s'est forgé une place dans l'esthétique pop du monde entier et a engendré une vision romantique de la culture aborigène » (p. 241).

"L'enseignement de la world music en Occident» (p. 246-265) est étudié par Trevor Wiggins. Ce dernier montre les aspects positifs de cette entreprise, mais signale qu'elle reste très limitée. Tout en permettant à des étudiants de se pencher sur des questions relatives au multiculturalisme ou de découvrir de nouveaux univers musicaux, cet enseignement est en " deçà de la réalité " (p. 254) et évolue tel un outil politique pour régler des problèmes interculturels. Malgré un chapitre consacré à la définition et à la terminologie du concept de world music, l'auteur reste vague quant au sens précis de ce terme. Renvoie-t-il aux musiques traditionnelles non occidentales, aux compositions syncrétiques ou aux deux à la fois?

De son côté, Line Grenier ("Circulation, valorisation et localisation de la musique pop planétaire : le cas de Céline Dion », p. 286-312) s’intéresse à l'émancipation des musiques populaires à travers le monde. Elle réalise une analyse pointilleuse de la célébrité de Céline Dion, cas à partir duquel elle renverse de nombreux a priori et préjugés comme la conception d'une hiérarchisation dans les musiques, notamment l'idée que la pop planétaire est une grande menace pour la mondialisation et qu'elle va à l'encontre de l'intégrité des cultures. Elle affirme que le succès planétaire des musiques populaires n'est pas uniquement commercial. Elle en conclut alors que « le succès et l'estime ou le dénigrement naissent des processus interconnectés de circulation, valorisation et localisation qui servent d'intermédiaires à la mise en société (Hennion, 1993) de la musique populaire» (p. 293).

Jocelyne Guilbault, dans un texte qui clôture cette première partie (« Mondialisation et localisme », p. 313-334), s'adonne à une synthèse diachronique de la problématique de la circulation du musical, notion qu'elle ramène à celle de mondialisation. Cet article théorique examine «la façon dont les discours ethnomusicologiques ont exposé le phénomène [...] et, ce faisant, construit le concept de localisme » (p. 314). De cette réflexion découle l'idée que le local est un produit de l'histoire, tout comme la mondialisation associée à l'occidentalisation. "Le local est cet autre construit à travers le filtre d'une perspective occidentale qui pense a priori que celui-ci existe à l'état pur avant l'arrivée des Occidentaux » (p. 316). À partir de cette observation, elle cherche à déboulonner les préjugés sur la pureté des musiques non occidentales. En ce sens, l'argumentation développée ici incitera peut-être les puristes à s'interroger davantage sur les conséquences du contact au fondement du phénomène musical, ainsi que sur l'interprétation qu'on peut en faire.

Plusieurs des articles de cette première partie de "L'Unité de la musique » sont consacrés à des sujets souvent négligés par la musicologie traditionnelle, tels les phénomènes musicaux d'hybridité ou les musiques populaires. Tout ce qui ne relève pas d'un phénomène de pureté a souvent été dévalorisé, voire délaissé, tant par des ethnomusicologues qui ont cherché une forme d'idéalisme dans des cultures affranchies de tout contact, que par des musicologues qui 
ne jugent que par l'intermédiaire des musiques dites savantes. Cette partie est donc une contribution majeure à une musicologie générale.

Après avoir dressé un panorama de la diversité musicale actuelle, Nattiez nous propose d'examiner les grands traits qui caractérisent la spécificité et la parenté des cultures musicales. De cette proposition découlent les questions centrales de typologies et d'universaux auxquelles Molino et Nattiez vont consacrer un article d'une cinquantaine de pages qui motive à lui seul la naissance de ce projet encyclopédique ("Typologies et universaux», p. 337-396). En partant de la métaphysique pour se diriger vers les sciences humaines, les auteurs nous offrent un historique détaillé de ces questions, puis dressent un inventaire des arguments contre et pour la recherche des universaux. C'est en soumettant la diversité des formes culturelles aux lois physiques, perceptives et cognitives de l'espèce humaine qu'ils jugent nécessaire de repenser la question des universaux, trop longtemps écartée selon eux des préoccupations scientifiques des musicologues. Par cet essai, une solution pour repenser les méthodes du passé est envisagée. Celle-ci est fondée sur l'élaboration de typologies construites à partir de catégorisations pertinentes, lesquelles s'en remettent à la théorie du prototype de Wittgenstein.

La recherche d'unité et d'universaux rencontre son apogée lorsque Bruno Nettl, Marcello Sorce Keller et Jean Molino proposent une typologie de notions majeures de l'étude du musical. En examinant « la notion d'authenticité dans les musiques occidentales et non occidentales » (p. 1113-1126), Bruno Nettl s'interroge sur la réelle pertinence de cette question. Selon l'auteur, la notion d'authenticité a trop longtemps été utilisée comme valeur de jugement d'une exécution, plutôt que comme critère d'analyse; elle doit aujourd'hui être mise en relation avec l'histoire des musiques et leur possible renouvellement. Dans cette perspective, la notion d'authenticité serait liée à celle d'identité à laquelle Marcello Sorce Keller s'attaque ("Représentation et affirmation de l'identité dans les musiques occidentales et non occidentales », p. 1127-1153). Keller nous dit que ce sentiment d'appartenance est universel, mais non " monolithique " : "L'identité que nous appelons 'ethnique' est un fait social pour ainsi dire 'biodégradable’ et, donc, non irréductible » (p. 1136). Ainsi, tout en montrant l'universalité de la musique-qui réside dans le fait qu'elle manifeste une forte capacité à caractériser des groupes humains -, on constate que les stratégies de caractérisation du sentiment d'identité sont plurielles.

Quant à Jean Molino, dans son article « Du plaisir à l'esthétique : les multiples formes de l'expérience musicale » (p. 1154-1196), il complique la réflexion en y ajoutant la question de l'esthétique. Fidèle à sa théorie sur la réception de la musique, il procède à une typologie des expériences musicales, terme qu'il préfère à celui d'esthétique. Pour Molino, la musique est face à une multiplicité de réactions et de comportements qui constituent un défi dans l'élaboration d'une théorie générale du goût. En insistant sur la diversité des plaisirs et sur la complexité de la dynamique de l'expérience musicale, il s'accorde pour dire que cette multiplicité est organisée et qu'on retrouve des phylums communs de l'expérience musicale entre toutes les cultures. 
La question posée ici est essentielle et avec Charles Landesman (1971, 13), l'on peut constater que la théorie des universaux nie le critère d'identité propre à chaque objet que l'on considère comme universel. Tout comme l'a suggéré Platon jadis, la question des universaux n'est pas fondée sur la perception mais sur la raison : les idées existent comme des réalités supérieures (voir Landesman 1971, 16). Si la théorie des universaux est séduisante, elle reste en même temps très théorique et trop éloignée de la réalité perceptive pour qu'elle puisse combler les attentes en tant que modèle scientifique. Et bien que les universaux répondent à nos interrogations sur le comportement humain, comment expliquer le particulier, l'individuel et le singulier?

Foncièrement positiviste et post-structuraliste, Nattiez reproche au culturalisme d'être " réducteur et déterministe » (p. 18). Quant au relativisme, il l'aborde pour une première fois dans le contexte du développement postmoderne de la musicologie, entre autres avec l'arrivée de la New Musicology à la fin des années 1980 (p. 21-23). Dans son article conclusif (en pages 1207 et 1208), il expose clairement sa vision des faits sur l'effet néfaste du relativisme et la nécessité d'un discours musicologique " gouverné par l'exigence de vérité, même si, comme [il l'a] proposé ailleurs (Nattiez, 1993), il s’agit de vérités locales " (p. 1207-1208).

Sans ce relativisme, n'est-ce pas une société élitiste, voire univoque et renonçant à toute forme d'idéologies divergentes qui se développe ? La quête de vérité et d'universaux renvoie de façon indirecte au pouvoir. Lorsqu'on est en possession du vrai, il nous semble qu'on exerce une autorité sur le faux, et cette autorité se traduit par l'exercice de pouvoir réalisable lorsqu'elle est sujette à une reconnaissance formelle et implicite. Dans la recherche de vérités et d'universaux, nous sommes dans l'attente d'une reconnaissance extérieure afin d'exercer un pouvoir sur ce qui sera considéré comme faux.

Tout récemment, le sociologue Alvin Toffler, dans La richesse révolutionnaire, a remis en question le concept de vérité. À partir d'un bref historique de l'évolution des connaissances de Platon à nos jours, il montre que les idées et les hypothèses sont rendues fausses par le temps, car « chaque fragment de connaissance a une durée de vie limitée " $(2007,164)$. Les idées et les théories sont dépassées par l'évolution du monde ou remplacées par des vérités postérieures, censées être plus exactes. Il explique également que les analogies et les classifications que nous réalisons pour identifier des similitudes entre des phénomènes ou encore pour tirer des conclusions de vérité, sont épineuses. De par l'évolution du monde, «d'anciennes similitudes peuvent se transformer en dissemblances, et des comparaisons perdre de leur évidence» $(2007,168)$. L'approche de Toffler nous force à tenir compte du fait que la question de la vérité et des universaux s'essouffle face à l'évolution rapide de nos sociétés actuelles.

\section{EN GUISE DE CONCLUSION}

La couverture du cinquième volume d'Une Encyclopédie pour le XXI siècle représente le duo formé des musiciens américain et indien Yehudi Menuhin et Ravi Shankar, respectivement au violon et au sitar. Cette photo-illustrant un 
concert aux États-Unis réalisé pour le compte de l'ONU lors du jour de la célébration des Droits de l'Homme en 1967-suggère la synthèse entre les cultures occidentale et orientale. Pourtant, en tenant compte du fait que Jean-Jacques Nattiez cherche à éliminer les a priori concernant les musiques savantes et populaires, on pourrait s'interroger sur le choix de cette couverture. Malgré la coexistence de ces deux musiciens issus de cultures différentes, les deux artistes ont un point en commun, non négligeable et qui prend tout son sens dans la hiérarchie sociale : ils font partie de la sphère de la musique dite savante ! Mais peut-être est-ce une autre manière de montrer qu'à travers la diversité on peut retrouver l'unité ?

Malgré quelques réserves exprimées quant au fardeau théorique qu'on cherche à lui faire porter, ce cinquième volume clôture brillamment l'entreprise encyclopédique entamée en 2001 par Jean-Jacques Nattiez et ses collaborateurs, soit Margaret Bent, Rossana Dalmonte et Mario Baroni. La politique éditoriale de l'ensemble du projet permet au lecteur de découvrir des travaux de recherche de divers horizons, où se confrontent des idéologies distinctes et contradictoires. Ce projet encyclopédique est l'œuvre d'une audacieuse fusion de sujets, d'objets et d'approches du fait musical. Son ingéniosité tient à la fois au contenu des articles et aux liens qui s'établissent entre eux. Détournant le parcours habituel des autres encyclopédies classiques en misant sur la diversité d'opinions, ces cinq volumes nous livrent une richesse scientifique inestimable.

Ce dernier volume nous conduit vers une nouvelle approche de la musicologie et de l'étude du musical. Malgré les réserves émises sur cette démarche, notamment en ce qui concerne la quête d'universaux, d'unité et de vérité, nous voudrions insister sur cette audacieuse entreprise qui réussit l'exploit d'analyser le fait musical tant d'un point de vue de l'objet, des processus que des enjeux (Desroches, 2005). De plus, ce dernier volume ouvre un espoir en direction des générations à venir qui, nous l'espérons, tendront vers l'échange, l'écoute, le partage et l'intérêt pour chacun des savoirs, des approches et des sonorités liés au fait musical. La richesse des informations contenues dans ce volumetout comme dans les quatre précédents-entraînera très loin le lecteur curieux dans sa réflexion sur le Musical.

\section{RÉFÉRENCES}

Desroches, Monique. 2005. "Musique et rituel : signification, identité et société ». Musiques. Une Encyclopédie pour le XXI siècle, "3. Musiques et cultures », sous la dir. de Jean-Jacques Nattiez, 538-556. Arles-Paris : Actes Sud/Cité de la musique.

Hennion, Antoine. 1993. La passion musicale. Paris : Métailié.

Landesman, Charles. 1971. The Problem of Universals. New-York: Basic Books. Menger, Pierre-Michel. 2001. Le paradoxe du musicien. Paris : L'Harmattan. Nattiez, Jean-Jacques. 1993. Le combat de Chronos et d'Orphée. Paris : Christian Bourgois.

Seeger, Charles. 1960. "On the Moods of a Musical Logic ». Journal of the American Musicological Society XIII, $\mathrm{n}^{\mathrm{O}}$ 1-3 : 224-261. Repris dans 1977. Studies in Musicology 1935-1975, 64-88. Berkeley : University of California Press. 
Toffler, Alvin, 2007. La richesse révolutionnaire. Paris : Plon.

Théberge, Paul. 1997. Any Sound You Can Imagine: Making Music/Consuming Technology. Middletown : Wesleyan University Press.

Wittgenstein, L. J. 1953. Philosophische Untersuchungen-Philosophical Investigations. Oxford : Blackwell.

JESSICA RoDA

Didier van Moere. 2008. Karol Szymanowski. Paris : Fayard. 695 p. ISBN 978-2213-63774-7 (couverture cartonnée).

"J'aime ce qui est délaissé ». C'est en commençant cette biographie par une citation de Vladimir Jankélévitch (tirée d'un entretien radiophonique) que Didier van Moere rappelle à notre mémoire (p. 9) l'importance de ce compositeur polonais (1882-1937). Ce livre, qui vient tout juste de paraître dans la collection des éditions Fayard destinée aux biographies de musiciens, offre un panorama très complet de la vie, de l'œuvre et de la pensée de cet héritier de Chopin. Il s'agit ici d'un ouvrage de référence indispensable pour quiconque s'intéresse à ce compositeur, ou encore, de manière plus générale, à la musique polonaise du début du $\mathrm{XX}^{\mathrm{e}}$ siècle, ou même aux musiques étrangères présentes à Paris à cette époque. Rappelons à cet effet que Szymanowski a participé de façon très active au milieu musical français, comme le mentionne Michel Duchesneau, notamment en s'associant régulièrement aux activités de la SMI : « Milhaud ne cesse de réclamer que l'on joue les œuvres de Szymanowski, car il voit en ce compositeur un représentant de tout premier ordre de la musique moderne » (1997, p. 160).

Un ouvrage exhaustif portant sur ce compositeur manquait grandement à la littérature musicologique. Nous n'avions jusqu'à présent que de rares biographies, malheureusement incomplètes et qui remontent aux années 1990 (notamment celles de Christopher Palmer (1987), et de Jim Samson (1990)).

Agrégé de lettres classiques et docteur en musicologie, Didier van Moere, auteur d'une thèse sur Szymanowski et la France, soutenue à l'Université Paris Sorbonne (Paris IV) en 1993, enseigne maintenant les rapports entre la littérature et la musique à l'Université Stendhal-Grenoble 3 en France. Il a entrepris, pour la publication de ce livre, le dépouillement de fonds d'archives gigantesques, tels ceux de la bibliothèque universitaire de Varsovie, la correspondance générale de Szymanowski et l'ensemble de ses écrits, musicaux et littéraires.

La vie de Szymanowski est présentée dans l'ordre chronologique. La présentation acquiert un grand intérêt du fait que les éléments biographiques sont entrecoupés par les analyses musicales, génétiques et stylistiques des œuvres de Szymanowski. Ces descriptions des œuvres sont présentées en retrait, dans le texte, et dans une police différente, ce qui rend la lecture très aisée.

À travers les onze chapitres qui constituent le livre, van Moere trace un portrait très utile de la situation sociopolitique de l'époque que traverse le protagoniste. 